\title{
Bound-state dark matter and Dirac neutrino masses
}

\author{
M. Reig, ${ }^{1, *}$ D. Restrepo, ${ }^{2, \dagger}$ J. W. F. Valle, ${ }^{1, \star}$ and O. Zapata ${ }^{2, \S}$ \\ ${ }^{1}$ AHEP Group, Institut de Física Corpuscular-C.S.I.C./Universitat de València, \\ Parc Cientific de Paterna. C/Catedratico José Beltrán, 2 E-46980 Paterna (València), Spain \\ ${ }^{2}$ Instituto de Física, Universidad de Antioquia, Calle 70 No. 52-21, \\ Apartado Aéreo 1226, Medellín, Colombia
}

(Received 13 March 2018; published 19 June 2018)

\begin{abstract}
We propose a simple theory for the idea that cosmological dark matter (DM) may be present today mainly in the form of stable neutral hadronic thermal relics. In our model, neutrino masses arise radiatively from the exchange of colored DM constituents, giving a common origin for both dark matter and neutrino mass. The exact conservation of $B-L$ symmetry ensures dark matter stability and the Dirac nature of neutrinos. The theory can be falsified by dark matter nuclear recoil direct detection experiments, leading also to possible signals at a next generation hadron collider.
\end{abstract}

DOI: $10.1103 /$ PhysRevD.97.115032

\section{INTRODUCTION}

The common lore concerning particle dark matter candidates has long been that they must be electrically neutral and carry no color. This view has been challenged by the authors in Ref. [1], who suggested that dark matter (DM) may be the lightest hadron made of two stable color octet Dirac fermions $\mathcal{Q}$ with mass below $10 \mathrm{TeV}$. The interest in this idea may go well beyond QCD, since analogous bound-state DM candidates emerge in models with a new confining hypercolor interaction [2].

We argue that neutrino mass and cosmological dark matter may have a common origin, with the underlying DM physics acting as messenger of neutrino mass generation [3-6]. We propose a simple implementation of the bound-state dark matter scenario in which DM constituents induce calculable neutrino mass at the radiative level. Dark matter is a QCD bound state $\mathcal{Q} \mathcal{Q}$ stabilized by the same conserved $B-L$ symmetry associated to the Dirac nature of neutrinos [7].

In addition to the heavy Dirac fermion, our model introduces extra scalars, in order to ensure that at least two neutrino masses are nonzero, required by the neutrino oscillation data in order to account both for solar and atmospheric mass scales $[8,9]$. This simple picture can account for current neutrino oscillation and dark matter

\footnotetext{
*mario.reig@ific.uv.es

restrepo@udea.edu.co

*valle@ific.uv.es, URL: de http://astroparticles.es/

§oalberto.zapata@udea.edu.co
}

Published by the American Physical Society under the terms of the Creative Commons Attribution 4.0 International license. Further distribution of this work must maintain attribution to the author(s) and the published article's title, journal citation, and DOI. Funded by SCOAP. phenomena, and can be falsified relatively soon, in nuclear recoil studies at XENON1T [10]. Moreover, the extra colored states, including scalar bosons, may lead to new phenomena at a next generation hadron collider.

\section{THE MODEL}

As a theory preliminary, we recall that, within the type-I seesaw mechanism with a single right-handed neutrino, two neutrinos remain massless after the seesaw [11]. This degeneracy is lifted by calculable loop corrections. ${ }^{1}$ Here we propose a variant radiative seesaw scheme, in which a single colored fermion Dirac messenger $\mathcal{Q}$ is introduced, charged under the $U(1)_{B-L}$ symmetry, plus two sets of colored scalars, labeled by $a=1,2$; see Table I.

Apart from the right-handed neutrinos, all of the new particles are colored. For definiteness, we assign them to the octet $S U(3)_{c}$ representation. ${ }^{2}$ We also impose that the $B-L$ symmetry holds, together with a $Z_{2}$ symmetry. The former ensures that $\mathcal{Q}$ has only a Dirac-type mass term, while the latter forbids the tree-level Dirac neutrino mass terms from $\overline{\nu_{R i}} \tilde{H}^{\dagger} L_{j}$. The Lagragian contains the following new terms (summation is implied over repeated indices, and trace over $\mathbf{N}_{c}=\mathbf{8}$ is implicit.)

$$
\begin{aligned}
\mathcal{L} \supset & -\left[h_{i}^{a} \overline{\mathcal{Q}_{R}} \tilde{\eta}_{a}^{\dagger} L_{i}+M_{\mathcal{Q}} \overline{\mathcal{Q}_{R}} \mathcal{Q}_{L}+y_{i}^{a} \overline{\nu_{R i}} \sigma_{a}^{*} \mathcal{Q}_{L}+\text { H.c. }\right] \\
& -\mathcal{V}\left(H, \eta_{a}, \sigma_{a}\right) .
\end{aligned}
$$

\footnotetext{
${ }^{1}$ An analogous situation happens in supersymmetry with bilinear breaking of $\mathrm{R}$ parity [12-14], which induces the solar scale at one-loop, once atmospheric scale is taken as tree level input.

${ }^{2}$ Notice, however, that our neutrino mass discussion also holds if they had different $S U(3)_{c}$ transformation properties.
} 
TABLE I. Left-handed fermions and scalars. When the vectorlike quark $\mathcal{Q}$ is taken as a color octet, $\mathbf{N}_{c}=\mathbf{8}$, one can realize bound-state dark matter scenario proposed in Ref. [1].

\begin{tabular}{lccr}
\hline \hline Particles & $U(1)_{B-L}$ & $\left(\mathrm{SU}(3)_{c}, \mathrm{SU}(2)_{L}\right)_{Y}$ & $Z_{2}$ \\
\hline$Q_{i}=\left(u_{L} d_{L}\right)_{i}^{T}$ & $+1 / 3$ & $(\mathbf{3}, \mathbf{2})_{1 / 6}$ & + \\
$\overline{u_{R i}}$ & $-1 / 3$ & $(\overline{\mathbf{3}}, \mathbf{1})_{-2 / 3}$ & + \\
$\overline{d_{R i}}$ & $-1 / 3$ & $(\overline{\mathbf{3}}, \mathbf{1})_{1 / 3}$ & + \\
$L_{i}=\left(\nu_{L} e_{L}\right)_{i}^{T}$ & -1 & $(\mathbf{1}, \mathbf{2})_{-1 / 2}$ & + \\
$\overline{e_{R i}}$ & +1 & $(\mathbf{1}, \mathbf{1})_{1}$ & + \\
$\overline{\nu_{R i}}$ & +1 & $(\mathbf{1}, \mathbf{1})_{0}$ & - \\
$\mathcal{Q}_{L}$ & $-r$ & $\left(\mathbf{N}_{c}, \mathbf{1}\right)_{0}$ & + \\
$\overline{\mathcal{Q}_{R}}$ & $r$ & $\left(\mathbf{N}_{c}, \mathbf{1}\right)_{0}$ & + \\
$H$ & 0 & $(\mathbf{1}, \mathbf{2})_{1 / 2}$ & + \\
$\sigma_{a}$ & $1-r$ & $\left(\mathbf{N}_{c}, \mathbf{1}\right)_{0}$ & - \\
$\eta_{a}$ & $1-r$ & $\left(\mathbf{N}_{c}, \mathbf{2}\right)_{1 / 2}$ & + \\
\hline \hline
\end{tabular}

The condition $r \neq 1$ forbids Higgs-like Yukawa couplings of $\eta^{\alpha}$ to the Standard Model fermions (for $r=1$ one would need an additional $Z_{2}$ symmetry, as in [15]). The new part of the scalar potential can be cast as

$$
\begin{aligned}
\mathcal{V}\left(H, \eta_{a}, \sigma_{a}\right)= & \mathcal{V}\left(\eta_{a}\right)+\mathcal{V}\left(\sigma_{a}\right)+\mathcal{V}\left(\eta_{a}, \sigma_{a}\right) \\
& +\mathcal{V}\left(H, \eta_{a}\right)+\mathcal{V}\left(H, \sigma_{a}\right)
\end{aligned}
$$

where the various terms in the Higgs potential are

$$
\begin{aligned}
& \mathcal{V}\left(\eta_{a}, \sigma_{a}\right)= \kappa^{a b} \operatorname{Tr}\left(\sigma_{a} \eta_{b}^{\dagger}\right) H+\lambda_{\sigma \eta}^{a b c d} \operatorname{Tr}\left(\sigma_{a}^{*} \sigma_{b}\right) \operatorname{Tr}\left(\eta_{c}^{\dagger} \eta_{d}\right) \\
&+\tilde{\lambda}_{\sigma \eta}^{a b c d} \operatorname{Tr}\left(\sigma_{a}^{*} \eta_{b}\right) \operatorname{Tr}\left(\sigma_{c} \eta_{d}^{\dagger}\right)+\text { H.c., } \\
& \mathcal{V}\left(H, \eta_{a}\right)= \lambda_{3 \eta H}^{a b}\left(H^{\dagger} H\right) \operatorname{Tr}\left(\eta_{a}^{\dagger} \eta_{b}\right)+\lambda_{4 \eta H}^{a b} \operatorname{Tr}\left[\left(\eta_{a}^{\dagger} H\right)\left(H^{\dagger} \eta_{b}\right)\right] \\
&+ \text { H.c., } \\
& \mathcal{V}\left(H, \sigma_{a}\right)=\lambda_{\sigma H}^{a b} \operatorname{Tr}\left(\sigma_{a}^{*} \sigma_{b}\right) H^{\dagger} H+\text { H.c.. }
\end{aligned}
$$

Since $C P$ conservation is assumed, the $C P$-even and $C P$-odd scalars do not mix. Moreover, terms like $\left(\eta^{\dagger} H\right)^{2}$ are also forbidden and, as a consequence, the real and imaginary parts of the scalars with nonzero $B-L$ charges are degenerate. Note, however, that the cubic scalar coupling terms $\kappa^{a b}$ breaking the $Z_{2}$ symmetry softly allow for the mixing between the $\sigma_{a}$ and $\eta_{a}$ [16]. At the end, the $4 \times 4$ mass matrices for the $C P$-odd and $C P$-even scalars are equal, since the $\kappa^{a b}$ terms do not break such a degeneracy.

In order to illustrate the neutrino mass generation mechanism, we consider the following block-diagonal mass matrix for the $C P$-even scalars (in the basis $S_{R}=$ $\left.\left(\eta_{1 R}^{0}, \sigma_{1 R}^{0}, \eta_{2 R}^{0}, \sigma_{2 R}^{0}\right)^{T}\right)$ :

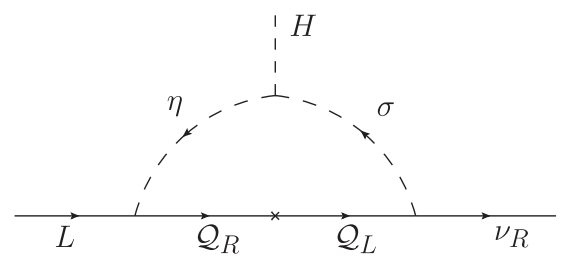

FIG. 1. $B-L$ flux in the one-loop Dirac neutrino mass.

$$
\mathcal{M}_{R}^{2}=\left(\begin{array}{cccc}
\left(\mu_{\eta}^{11}\right)^{2} & \frac{\kappa_{11} v}{\sqrt{2}} & 0 & 0 \\
\frac{\kappa_{11} v}{\sqrt{2}} & \left(\mu_{\sigma}^{11}\right)^{2} & 0 & 0 \\
0 & 0 & \left(\mu_{\eta}^{22}\right)^{2} & \frac{\kappa_{22} v}{\sqrt{2}} \\
0 & 0 & \kappa_{22} \frac{v}{\sqrt{2}} & \left(\mu_{\sigma}^{22}\right)^{2}
\end{array}\right) .
$$

Here we have used the parametrization $\eta_{a}=\left(\eta_{a}^{+},\left(\eta_{a R}^{0}+\right.\right.$ $\left.\left.i \eta_{a I}^{0}\right) / \sqrt{2}\right)^{T}, \sigma_{a}=\left(\sigma_{a R}^{0}+i \sigma_{a I}^{0}\right) / \sqrt{2}$ and $H=(0,(h+v) /$ $\sqrt{2})^{T}$, with $h$ denoting the standard model Higgs boson, and $v=246 \mathrm{GeV}$. The parameters $\mu_{\eta(\sigma)}^{a b}$ are the quadratic mass terms after electroweak symmetry breaking in $\mathcal{V}\left(\eta_{a}\right)$ and $\mathcal{V}\left(\sigma_{a}\right)$.

Since the tree-level Dirac mass term is forbidden by symmetry, calculable neutrino masses are generated at one-loop order, by the Feynman diagram displayed in Fig. 1. One finds the following effective mass matrix

$$
\begin{aligned}
\left(\mathcal{M}_{\nu}\right)_{i j}= & N_{c} \frac{M_{\mathcal{Q}}}{64 \pi^{2}} \sum_{a=1}^{2} h_{i}^{a} y_{j}^{a} \frac{\sqrt{2} \kappa_{a a} v}{m_{S_{2 R}^{a}}^{2}-m_{S_{1 R}^{a}}^{2}} \\
& \times\left[F\left(\frac{m_{S_{2 R}^{a}}^{2}}{M_{\mathcal{Q}}^{2}}\right)-F\left(\frac{m_{S_{1 R}^{a}}^{2}}{M_{\mathcal{Q}}^{2}}\right)\right]+(R \rightarrow I)
\end{aligned}
$$

where $F\left(m_{S_{\beta}}^{2} / M_{Q}^{2}\right)=m_{S_{\beta}}^{2} \log \left(m_{S_{\beta}}^{2} / M_{Q}^{2}\right) /\left(m_{S_{\beta}}^{2}-M_{Q}^{2}\right)$ and the $S U(3)_{c}$ color factor $N_{c}$ is assumed to be 8 , since the new particles running in the loop transform as octets. The four $C P$-even mass eigenstates are denoted as $S_{1 R}^{1}, S_{2 R}^{1}, S_{1 R}^{2}, S_{2 R}^{2}$, with a similar notation for the $C P$-odd ones. Notice that the above effective neutrino mass matrix in does not have the projective rank one structure $\left(\mathcal{M}_{\nu}\right)_{i j}^{\text {tree-level }} \propto h_{i}^{a} y_{j}^{b}$ characterizing the Dirac variant [7] of the "incomplete" tree-level seesaw mechanism, involving the exchange of a single heavy messenger, called $(3,1)$ in Ref. [11]. ${ }^{3}$

Here the effective one-loop induced neutrino mass matrix has in general rank two, implying two nonvanishing Dirac neutrino masses. As a simple numerical estimate, let's consider the case $\mu_{\eta}^{a a}=\mu_{\sigma}^{a a} \gg \sqrt{2} \kappa^{a a} v$. Taking $m_{S_{2 R}^{a}}^{2}-$ $m_{S_{1 R}^{a}}^{2}=\sqrt{2} \kappa^{a a} v$ and $m_{S_{2 R}^{a}}^{2}+m_{S_{1 R}^{a}}^{2}=2\left(\mu_{\eta}^{a a}\right)^{2}$ one finds

\footnotetext{
${ }^{3}$ Here one would recover the rank-one situation characterizing the type-I seesaw in the limit where one set of scalars decouples.
} 


$$
\begin{aligned}
\left(\mathcal{M}_{\nu}\right)_{i j}= & N_{c} \frac{M_{\mathcal{Q}}}{32 \pi^{2}} \sqrt{2} \kappa^{a a} v \sum_{a=1}^{2} \frac{h_{i}^{a} y_{j}^{a}}{\left(\mu_{\eta}^{a a}\right)^{2}-M_{\mathcal{Q}}^{2}} \\
& \times\left[1-\frac{M_{\mathcal{Q}}^{2}}{\left(\mu_{\eta}^{a a}\right)^{2}-M_{\mathcal{Q}}^{2}} \log \left(\frac{\left(\mu_{\eta}^{a a}\right)^{2}}{M_{\mathcal{Q}}^{2}}\right)\right],
\end{aligned}
$$

so that if $\left(\mu_{\eta}^{a a}\right)^{2} \gg M_{\mathcal{Q}}^{2}$ one has

$$
\begin{aligned}
\left(\mathcal{M}_{\nu}\right)_{i j}= & N_{c} \frac{M_{\mathcal{Q}}}{32 \pi^{2}} \sqrt{2} v \sum_{a=1}^{2} \kappa^{a a} \frac{h_{i}^{a} y_{j}^{a}}{\left(\mu_{\eta}^{a a}\right)^{2}} \\
\sim & 0.03 \mathrm{eV}\left(\frac{M_{\mathcal{Q}}}{9.5 \mathrm{TeV}}\right)\left(\frac{\kappa^{a a}}{1 \mathrm{GeV}}\right) \\
& \times\left(\frac{50 \mathrm{TeV}}{\mu_{\eta}^{a a}}\right)^{2}\left(\frac{h_{i}^{a} y_{j}^{a}}{10^{-6}}\right) .
\end{aligned}
$$

One sees that, indeed, small neutrino masses arise naturally by taking reasonable values for the Yukawa couplings, small value for the soft breaking parameter $\kappa^{a b}$, as well as sufficiently large values for the scalar masses. Notice that the smallness of $\kappa^{a b}$ is natural in t'Hooft's sense [17], as the theory attains a larger symmetry when $\kappa^{a b} \rightarrow 0$, i.e., the smallness of neutrino mass is symmetry protected. Indeed, the naturalness of scotogenic mass generation has been established explicitly in the literature; see, for example, Ref. [18].

In short, concerning neutrino mass generation, our model provides a colored variant of the one suggested in [15]. However, our proposal provides the simplest implementation of the radiative Dirac seesaw mechanism, where the dark matter stability is genuinely associated to the Dirac nature of neutrinos. In fact, in our model, the scalars are charged under B-L, so that the stabilizing symmetry invoked in [15] is not required. Moreover, although the neutrino mass generation is similar in both models, the details of the associated physics differ substantially.

\section{BOUND-STATE DARK MATTER STABILITY FROM DIRAC NEUTRINOS}

The Dirac nature of neutrinos may ensure dark matter stability, as suggested in [7]. Here we clone this idea with the proposal that dark matter may be present today mainly in the form of stable neutral hadronic thermal relics. For definiteness we assume DM is a neutral bound-state of colored constituents, such as $\mathcal{Q} \mathcal{Q}$, where $\mathcal{Q}$ is a vectorlike color octet isosinglet fermion. It was claimed that a necessary and suficient condition for dark matter stability in this case is the presence of a global $U(1)_{D}$ dark baryon number, under which the $\mathcal{Q}$ is charged [1]. In our present model construction, the role of such apparently ad hoc symmetry is played by the usual $B-L$ symmetry present in the Standard Model. In fact, in our model dark matter stability, and the Dirac nature of the exotic fermion $\mathcal{Q}$ and

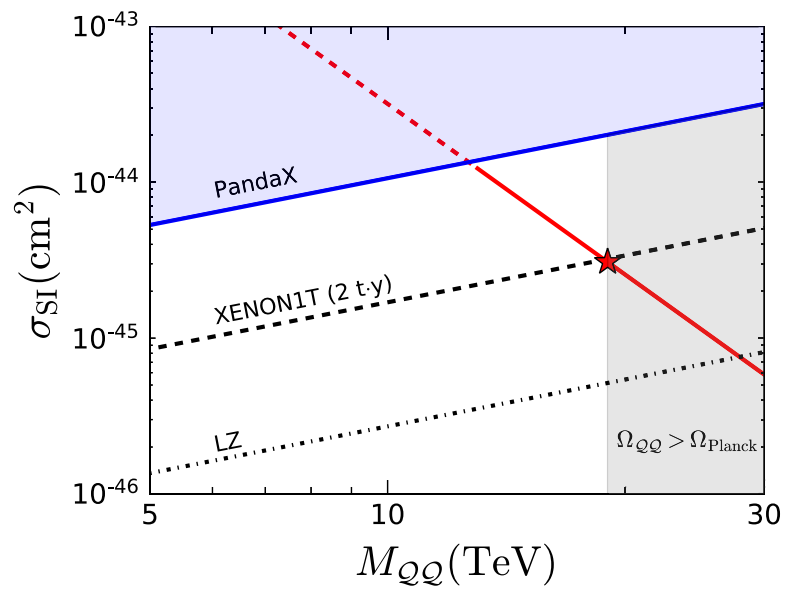

FIG. 2. The red line gives the spin independent cross section as a function of $M_{\mathcal{Q Q}}=2 M_{\mathcal{Q}}$. The star denotes the mass required for a thermal bound state DM (19 TeV). Smaller values can be probed by direct searches, the blue line gives the current bound, while the black (dashed, dotted and dot-dashed) lines represent future sensitivities.

of the neutrinos are all equivalent, and result from $B-L$ conservation.

Hence, by construction, the new Yukawa interactions in Eq. (1) do not affect the stability of our bound state dark matter, since the colored scalars are heavier than $\mathcal{Q}$, and $\mathcal{Q Q}$ annihilation processes mediated by $\eta, \sigma$, are all forbidden by the conserved $B-L$ symmetry.

An adequate thermal relic density of bound-state dark matter requires the lightest constituent vectorlike color octet Dirac fermion, $\mathcal{Q}$, to have a mass $\approx 9.5 \mathrm{TeV}$, so that the $\mathcal{Q} \mathcal{Q}$ hadron weighs approximately $19 \mathrm{TeV}$ [1].

The set of scalars $\eta^{a}, \sigma^{a}$ in Fig. 1 can be either neutral or charged under $B-L$, depending on the baryon number assignment. If $B-L$ neutral, both $\eta^{a}$ and $\sigma^{a}$ are expected to be unstable, decaying to quarks [19] and two gluons respectively $[20,21]$. In contrast, in the second case, if the lightest octet particle were a scalar, then it would be strictly stable.

Bound-state dark matter will impart nuclear recoil in underground dark matter search experiments. The spinindependent direct detection cross-section is given as

$$
\sigma_{\mathrm{SI}} \approx 2 \times 10^{-45} \mathrm{~cm}^{2}\left(\frac{20 \mathrm{TeV}}{M_{\mathcal{Q Q}}}\right)^{6} \frac{\Omega_{\mathcal{Q Q}}}{\Omega_{\text {Planck }}},
$$

sharply correlated to the dark matter mass $M_{\mathcal{Q} \mathcal{Q}}=2 M_{\mathcal{Q}}$, as shown in the red line in Fig. 2.

In this figure, the star corresponds to the case where our bound-state DM makes up 100\% of the cosmological dark matter. In the presence of an additional dark matter particle, such as the axion, bound-state dark matter masses below $19 \mathrm{TeV}$ can be envisaged, as indicated by the red line. In this case, their contribution to the relic density will be correspondingly smaller, while the spin-independent cross 
section would be correspondingly larger. The blue line represents the current limit of PandaX [22]. The black (dashed, and dot-dashed) lines represent the future sensitivities expected at XENON1T [10] and LZ [23]. On the other hand we note that, within the standard thermal cosmological scenario, DM masses above $19 \mathrm{TeV}$ are ruled out by current observations by the Planck collaboration [24] (gray band).

Notice that the current LHC limit of $2 \mathrm{TeV}$ (next section) implies that the cross section is always small enough so as to have the bound-state dark matter candidate reaching underground detectors. For more detailed discussion and general aspects of the cosmology of a stable colored relic, see [25].

\section{COLOR OCTETS AT HADRON COLLIDERS}

In our model, the messengers of neutrino mass generation are the colored constituents of bound-state dark matter. Given enough energy, the $\mathcal{Q}$ 's are copiously pair produced at hadron colliders, through the processes $q_{i} \bar{q}_{i} \rightarrow$ $\mathcal{Q} \overline{\mathcal{Q}}$ and $g g \rightarrow \mathcal{Q} \overline{\mathcal{Q}}$, and are expected to hadronize. In contrast to WIMP dark matter scenarios, which engender only missing-energy signals, the bound-state dark matter scenario gives rise to very visible signals at hadron colliders, as they can form either neutral or charged bound states [1], e.g., neutral hybrid states $\mathcal{Q} g$ (detected as neutral hadrons, presumably stable) or charged $\mathcal{Q} q \bar{q}^{\prime}$ states, or more exotic $\mathcal{Q} q q q$ states, expected to be long-lived on collider time scales.

Current LHC data place a limit to the fermion color octet mass, $M_{\mathcal{Q}}>2 \mathrm{TeV}$ [26]. Since the cosmological relic abundance requires $M_{\mathcal{Q}} \approx 9.5 \mathrm{TeV}$, this scenario offers an attractive benchmark for future collider experiments beyond the energies attainable at the LHC. In fact, from the estimate in Ref. [27] one finds that a hadron collider of at least $65 \mathrm{TeV}$ center-of-mass energy would be required to probe the full cosmologically allowed range of masses of our bound-state DM model. This will allow a cross-check of the DM search results of XENON1T, expected quite soon, in just one year or so. Concerning the scalar messengers, we have two pairs of these, the $\sigma_{a} \sim(\mathbf{8}, 1,0)$, which are singlets under $S U(2)_{L}$, and the $\eta_{a}$, which transform as weak doublets, $\eta_{a} \sim$ $(\mathbf{8}, 2,1 / 2)$. As color octets, these would also be copiously produced at a hadron collider of sufficient energy [28]. However, their masses are expected to lie well above the reach of the LHC. Moreover, in our model these scalars carry nontrivial $B-L$ charges; see Table I. This makes them relatively inert with respect to the standard model fermions. This, in addition to their heavy masses, makes them very difficult to probe directly.

\section{LEPTON FLAVOR VIOLATION}

Our model may also lead to indirect virtual effects, such as charged lepton flavor violation. For example, the Yukawa interactions in Eq. (1) lead to radiative lepton

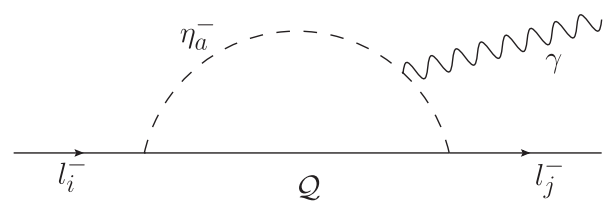

FIG. 3. Feynman diagram for the process $l_{i} \rightarrow l_{j} \gamma$.

flavor violation processes, as seen in Fig. 3, mediated by the charged scalar $\eta_{a}^{+}$.

The corresponding decay rate is given as [29]

$$
\begin{aligned}
\Gamma\left(l_{i} \rightarrow l_{j} \gamma\right)= & \frac{e^{2} m_{i}^{5}}{16 \pi}\left[\sum_{a} N_{c} h_{i}^{a} h_{j}^{a *} \frac{i}{16 \pi^{2} M_{\eta_{a}^{+}}^{2}}\right. \\
& \left.\times\left[\frac{-t^{2} \log t}{2(t-1)^{4}}+\frac{2 t^{2}+5 t-1}{12(t-1)^{3}}\right]\right]^{2},
\end{aligned}
$$

with $t=M_{\mathcal{Q}}^{2} / M_{\eta^{+}}^{2}$ and $N_{c}=8$. In the limit of heavy scalars, $t \rightarrow 0$, the decay width reads

$$
\Gamma\left(l_{i} \rightarrow l_{j} \gamma\right)=\frac{e^{2} m_{i}^{5}}{16 \pi}\left[\sum_{a} N_{c} h_{i}^{a} h_{j}^{a *} \frac{i}{16 \pi^{2} M_{\eta_{a}^{+}}^{2}}\left[-\frac{1}{12}\right]\right]^{2} .
$$

One sees that the current experimental constraint $\operatorname{BR}(\mu \rightarrow e \gamma)=\frac{\Gamma(\mu \rightarrow e \gamma)}{\Gamma_{\text {total }}}<5.7 \times 10^{-13}$, can be fulfilled provided

$$
\left(\sum_{a} \frac{h_{\mu}^{a} h_{e}^{a *}}{M_{\eta_{a}^{+}}^{2}}\right)^{2} \leq 5.7 \times 10^{-13} \frac{G_{F}^{2}}{\alpha_{\mathrm{EM}}} \frac{768 \pi}{N_{c}^{2}},
$$

which leads to a relatively mild requirement,

$$
\left|\sum_{a} h_{\mu}^{a} h_{e}^{a *}\left(\frac{50 \mathrm{TeV}}{M_{\eta_{a}^{+}}}\right)^{2}\right| \lesssim 1.5 .
$$

\section{SUMMARY AND OUTLOOK}

We have proposed a consistent viable theory for the recently proposed idea that the cosmological dark matter may be made up of stable colored relics forming neutral hadronic bound states of QCD. In our model, we have taken up at face value the suggestion in Ref. [1], employing an exotic vectorlike Dirac color octet fermion $\mathcal{Q}$ with mass below $10 \mathrm{TeV}$ as the dark matter constituent. In our construction, dark matter and neutrino mass generation both have a common origin. Our minimum particle content leads to two nonzero neutrino masses, associated to the solar and atmospheric scales. Bound-state dark matter stability is directly associated with the Dirac nature of neutrinos, and reflects the presence of an underlying exact $B-L$ symmetry. The scheme can account for both neutrino physics and dark matter phenomena, within a consistent ultraviolet complete setup, free of Landau poles up to the 
Planck scale, provided the scalars are heavy enough. Our model can be falsified relatively soon by dark matter searches, and could also be cross-checked later by a next generation hadron collider. Variants of our construction may be envisaged, in which the dark matter is bound by a new hypercolor interaction [30], instead of QCD, as suggested in Ref. [2].

\section{ACKNOWLEDGMENTS}

We thank Martin Hirsch for very useful discussions. Our work was supported by the Spanish Grants No. FPA201785216-P and No. SEV-2014-0398 (MINECO), Sostenibilidad-UdeA, and by COLCIENCIAS through Grants No. 11156584269 and No. 111577657253.
[1] V. De Luca, A. Mitridate, M. Redi, J. Smirnov, and A. Strumia, Colored dark matter, arXiv:1801.01135.

[2] M. Reig, J. W. F. Valle, C. A. Vaquera-Araujo, and F. Wilczek, A model of comprehensive unification, Phys. Lett. B 774, 667 (2017).

[3] E. Ma, Verifiable radiative seesaw mechanism of neutrino mass and dark matter, Phys. Rev. D 73, 077301 (2006).

[4] M. Hirsch et al., WIMP dark matter as radiative neutrino mass messenger, J. High Energy Phys. 1310 (2013) 149.

[5] A. Merle, M. Platscher, N. Rojas, J. W. F. Valle, and A. Vicente, Consistency of WIMP Dark Matter as radiative neutrino mass messenger, J. High Energy Phys. 07 (2016) 013.

[6] C. Bonilla, E. Ma, E. Peinado, and J. W. F. Valle, Two-loop Dirac neutrino mass and WIMP dark matter, Phys. Lett. B 762, 214 (2016).

[7] S. C. Chuliá, E. Ma, R. Srivastava, and J. W. F. Valle, Dirac neutrinos and dark matter stability from lepton quarticity, Phys. Lett. B 767, 209 (2017).

[8] P. F. de Salas et al., Status of neutrino oscillations 2017, arXiv:1708.01186.

[9] Valencia-Globalfit, http://globalfit.astroparticles.es/, 2018.

[10] E. Aprile et al. (XENON Collaboration), Physics reach of the XENON1T dark matter experiment, J. Cosmol. Astropart. Phys. 04 (2016) 027.

[11] J. Schechter and J. W. F. Valle, Neutrino masses in $\mathrm{SU}(2) \times$ U(1) theories, Phys. Rev. D 22, 2227 (1980).

[12] M. Hirsch, M. A. Díaz, W. Porod, J. C. Romão, and J. W. F. Valle, Neutrino masses and mixings from supersymmetry with bilinear $\mathrm{R}$ parity violation: A theory for solar and atmospheric neutrino oscillations, Phys. Rev. D 62, 113008 (2000); Erratum, Phys. Rev. D 65, 119901(E) (2002).

[13] M. A. Diaz, M. Hirsch, W. Porod, J. C. Romão, and J. W. F. Valle, Solar neutrino masses and mixing from bilinear R-parity broken supersymmetry: Analytical versus numerical results, Phys. Rev. D 68, 013009 (2003).

[14] M. Hirsch and J. W. F. Valle, Supersymmetric origin of neutrino mass, New J. Phys. 6, 76 (2004).

[15] Y. Farzan and E. Ma, Dirac neutrino mass generation from dark matter, Phys. Rev. D 86, 033007 (2012).
[16] F. Wilczek, Problem of Strong p and t Invariance in the Presence of Instantons, Phys. Rev. Lett. 40, 279 (1978).

[17] G. 't Hooft, Naturalness, chiral symmetry, and spontaneous chiral symmetry breaking, NATO Sci. Ser. B 59, 135 (1980).

[18] A. Merle and M. Platscher, Running of radiative neutrino masses: the scotogenic model-revisited, J. High Energy Phys. 11 (2015) 148.

[19] A. V. Manohar and M. B. Wise, Flavor changing neutral currents, an extended scalar sector, and the Higgs production rate at the CERN LHC, Phys. Rev. D 74, 035009 (2006).

[20] S. I. Bityukov and N. V. Krasnikov, The Search for new physics by the measurement of the four jet cross-section at LHC and FNAL, Mod. Phys. Lett. A A12, 2011 (1997).

[21] Y. Bai and B. A. Dobrescu, Collider tests of the renormalizable coloron model, J. High Energy Phys. 04 (2018) 114.

[22] X. Cui et al. (PandaX-II Collaboration), Dark Matter Results From 54-Ton-Day Exposure of PandaX-II Experiment, Phys. Rev. Lett. 119, 181302 (2017).

[23] D. S. Akerib et al. (LUX-ZEPLIN Collaboration), Projected WIMP sensitivity of the LUX-ZEPLIN (LZ) dark matter experiment, arXiv:1802.06039.

[24] P. A. R. Ade et al. (Planck Collaboration), Planck 2015 results. XIII. Cosmological parameters, Astron. Astrophys. 594, A13 (2016).

[25] M. Geller, S. Iwamoto, G. Lee, Y. Shadmi, and O. Telem, Dark quarkonium formation in the early universe, arXiv: 1802.07720 .

[26] V. Khachatryan et al. (CMS Collaboration), Search for heavy stable charged particles with $12.9 \mathrm{fb}^{-1}$ of 2016 data, Technical Report No. CMS-PAS-EXO-16-036, 2016.

[27] G. Grilli di Cortona, E. Hardy, and A. J. Powell, Dirac vs Majorana gauginos at a $100 \mathrm{TeV}$ collider, J. High Energy Phys. 08 (2016) 014.

[28] A. Hayreter and G. Valencia, LHC constraints on color octet scalars, Phys. Rev. D 96, 035004 (2017).

[29] L. Lavoura, General formulae for $f(1) \rightarrow f(2) \gamma$, Eur. Phys. J. C 29, 191 (2003).

[30] A. Mitridate, M. Redi, J. Smirnov, and A. Strumia, Dark matter as a weakly coupled dark baryon, arXiv: 1707.05380 . 